\title{
Musiques amplifiées : empreintes transculturelles dans le contexte franco-espagnol
}

Isabelle Marc Martínez

\section{CpenEdition}

1 Journals

Édition électronique

URL : https://journals.openedition.org/cher/11846

DOI : $10.4000 /$ cher.11846

ISSN : 2803-5992

Éditeur

Presses universitaires de Strasbourg

Édition imprimée

Date de publication : 1 décembre 2012

Pagination : 255-267

ISBN : 978-2-35410-049-0

ISSN : 1968-035X

\section{Référence électronique}

Isabelle Marc Martínez, "Musiques amplifiées : empreintes transculturelles dans le contexte francoespagnol », reCHERches [En ligne], 9 | 2012, mis en ligne le 14 février 2022, consulté le 16 février 2022. URL : http://journals.openedition.org/cher/11846; DOI : https://doi.org/10.4000/cher.11846

\section{(c) () (2)}

Ce(tte) œuvre est mise à disposition selon les termes de la Licence Creative Commons Attribution Pas d'Utilisation Commerciale - Partage dans les Mêmes Conditions 4.0 International. 


\section{Musiques amplifiées: empreintes transculturelles dans le contexte franco-espagnol}

Isabelle Marc Martínez

Département de Français - Universidad Complutense de Madrid

\section{Formes de la transculturalité dans les musiques amplifiées}

Quoi de plus français qu'Édith Piaf chantant «La foule»; quoi de plus américain que Frank Sinatra chantant «My way» ? Pourtant, l'on sait que ces chansons n'ont pas été créées à Paris ou à New York, mais ailleurs. Ainsi, la première reprend la mélodie de «Que nadie sepa mi sufrir »-composée par les Argentins Ángel Cabral (musique) et d'Enrique Dizeo (paroles) -, alors que la seconde se construit sur l'air de "Comme d'habitude» - musique et paroles de Claude François et Jacques Revaux. Ces deux produits emblématiques de la langue et de la culture françaises et américaines respectivement, par leur origine étrangère, illustrent les empreintes transculturelles caractérisant les musiques populaires contemporaines.

Ces musiques, également dénommées musiques amplifiées ou musiques actuelles, assimilables à la forme chanson, possèdent une dimension sociale et économique, mais aussi une dimension esthétique, en leur qualité de textes hybrides, multi-sémiotiques, où la musique, les paroles et la performance s'allient dans la construction d'un sens non premièrement pragmatique. Or, notamment depuis l'avènement des technologies de reproduction du son (Katz 2004), ces chansons, bien que produites et consommées sur un marché en principe local/national, ont tendance à dépasser les frontières et à devenir des produits transculturels dont les transferts sont régis par des logiques commerciales mais aussi culturelles et idéologiques. 
Les phénomènes liés à la mondialisation économique, l'intensification et la complexification des mouvements migratoires et la "cosmopolitisation " (Vertovec et Cohen 2002) des pratiques culturelles accentuent et intensifient les flux culturels en général et notamment les flux musicaux. C'est ainsi que la production et la consommation de musiques populaires se trouve de plus en plus marquée par ces transferts foisonnants et complexes. Or, à la différence d'autres produits culturels, l'étude de la transculturalité dans le domaine de la chanson doit tenir compte des différents langages à l'œuvre dans la construction du sens d'une chanson, à savoir, le langage musical (non-référentiel) et le langage verbal (référentiel) auxquels s'ajoute également le langage corporel/ performatif de l'interprète. En l'occurrence, «My way» et «La foule» sont des chansons incorporées à une culture cible par une reprise musicale, mais où les paroles originales - leur thématique y compris - ont été complètement supprimées en faveur d'un nouveau texte en langue vernaculaire. Ce type de reprise exclusivement musicale est l'une des formes possibles de transculturalité "chansonnière», allant de l'adaptation plus ou moins libre des paroles sur une même structure musicale à l'importation intégrale de chansons sur un nouveau marché, en passant par l'appropriation et l'imitation de styles, en langue vernaculaire ou étrangère. L'objectif du présent travail consiste à décrire ces transferts, notamment dans le contexte franco-espagnol.

Partons du transfert le plus évident, à savoir, la réception de chansons en version originale, sans altérations, dans une culture différente, assimilable donc à une véritable importation. En effet, partout dans le monde, à Paris, à Los Angeles, à Bangkok ou à Dakar, aujourd'hui, les médias (radio, télévision, Internet) diffusent des chansons importées de toutes pièces: on fredonne Lady Gaga et on danse sur les rythmes de David Guetta aux quatre coins de la planète. En ce qui concerne l'Espagne, actuellement, même si de nos jours la musique française a perdu une grande partie de son influence, un certain nombre d'artistes et de groupes français contemporains est apprécié par le public amateur et il existe même des émissions spécialement consacrées à la musique francophone (El Hexágono sur Radio 3 ou Nuestros Vecinos del Norte sur Radio 1). Par ailleurs, les «classiques» de la chanson (Brel, Aznavour, Piaf et d'autres encore) sont toujours diffusés sur les ondes et conservent leur prestige. De leur côté, quelques artistes et groupes espagnols ont bénéficié d'un bon accueil en France - Paco Ibáñez, Lluis Llach, Luz Casal, Mecano -, tout comme le flamenco et genres assimilables, qui connaissent un certain succès comme "musiques du monde». Néanmoins, en termes 
généraux, la musique populaire espagnole ${ }^{1}$ a une présence limitée sur le marché et la culture française contemporaine. Nous reviendrons plus tard sur les causes de cette présence moins importante des musiques amplifiées espagnoles en France et sur la prédominance de l'importation des musiques anglo-saxonnes dans les deux pays. Dans tous les cas, cependant, et même si les chansons importées sont reçues sans altérations musicales ni verbales, les publics, quant à eux, les transforment dans leur perception, conformément à leur propre culture et à la vision qu'en proposent les médias et les maisons de disques. Ainsi, «Ne me quitte pas» ne sera jamais perçue de la même façon en Belgique qu'en Australie, tout comme " $\mathrm{Al}$ alba» ne représentera jamais la même chose en Espagne qu'aux États-Unis. Cette lapalissade n'est pourtant pas sans conséquences, car elle souligne le caractère transculturel et non pas universel des musiques populaires, qui, même en gardant une même forme, sont perçues différemment en fonction des cultures.

Le deuxième mode de transfert consiste en la reprise de la structure musicale d'une chanson étrangère avec des paroles dans une langue cible qui ne possèdent aucun rapport avec le sens de l'original, comme dans «My way» ou «La foule». Dans ce cas, la composante culturelle originale est pratiquement éliminée de la reprise. Ainsi, «Que nadie sepa mi sufrir», dans la version de Piaf, subit une transformation substantielle: sur le plan verbal, les paroles en français traitent d'un sujet totalement différent à celles de la chanson en espagnol; sur le plan musical, si les notes sont conservées, les modifications du rythme et des arrangements remanient profondément la chanson. Ces altérations musicales et verbales, unies à la voix et à l'image d'Édith Piaf - la plus française des chanteuses françaises -, ont pour résultat un produit culturel totalement francisé. Il en est de même pour «My Way» qui, en dépit de son origine française, devient emblème de la culture populaire américaine. Ainsi, dans les reprises exclusivement musicales, l'empreinte de la culture source s'estompe à la faveur des transformations / adaptations opérées dans la culture de réception.

Troisièmement, des artistes locaux s'approprient les styles/esthétiques étrangers de façon plus ou moins directe. Il s'agit là d'un des phénomènes transculturels les plus reconnaissables et les plus prégnants, comme l'atteste, par exemple, le déferlement planétaire des musiques afro-américaines tout au long $\mathrm{du} \mathrm{xx}^{\mathrm{e}}$ siècle mais aussi la diffusion à l'échelle mondiale d'autres genres comme les musiques latines ou les musiques électroniques. Ainsi,

1 Les musiques latino-américaines constituent, logiquement, un phénomène beaucoup plus riche et complexe qu'il nous est impossible d'aborder dans ce travail. 
aussi bien à New York qu'à Cuenca, à Brest tout comme à Hong Kong, il est aujourd'hui possible d'écouter du rock, de la techno ou du hip hop avec des accents qui conjuguent le local et le mondial. En effet, l'ubiquité des musiques populaires constitue, sans doute, à la fois l'un des facteurs mais aussi l'un des effets les plus significatifs de la mondialisation dans les cultures populaires "glocales». Dans le contexte franco-espagnol, les «influences» sont complexes et bidirectionnelles (avec, toutefois, une majorité de transferts dans le sens France-Espagne). Pensons, par exemple, à la mode "andalouse» en France avec les Gipsy Kings ou Cathy Claret, ou au style "chanson» en Espagne, sur lequel nous reviendrons plus en détail, avec Paco Ibáñez ou Javier Krahe. L'appropriation stylistique/esthétique représente sans doute le mode le plus répandu d'acculturation musicale contemporaine, visible non seulement dans le domaine des musiques actuelles mais aussi traditionnellement dans celui des musiques savantes ${ }^{2}$.

La traduction et/ou adaptation de chansons constituerait la quatrième modalité de transfert dans le domaine qui nous occupe. À la fois reprise musicale et translation linguistique, ce processus transculturel est surtout visible actuellement dans la traduction/adaptation de comédies musicales américaines et anglaises, en version théâtrale ou cinématographique, doublées, sous-titrées ou surtitrées. Or, dans le contexte occidental des années 1950 et 1960 et plus tard encore, lorsque la mondialisation linguistique n'était pas encore un fait accompli, il était fréquent que les artistes publient des versions traduites dans les langues des différents pays récepteurs de leurs disques. Ainsi, lorsque Julio Iglesias, Mireille Matthieu, Petula Clark, Nat King Cole et bien d'autres ont voulu exporter leurs chansons, ils les ont chantées en version traduite. Par ailleurs, bien souvent des artistes locaux ont repris les tubes originaux dans la langue vernaculaire. Dans l'Espagne des années 1960, par exemple, la production de groupes comme les Mustang étaient presque uniquement consacrée à la reprise de succès étrangers; de leur côté, des stars étrangères (Aznavour, Celentano...) ont chanté leurs succès en espagnol. En France, de nombreux artistes, dont des vedettes comme Johnny Hallyday ou Eddie Mitchell, ont adapté ou repris, eux aussi, des «tubes» américains ou anglais. Dans ce genre d'adaptations il y avait souvent un intérêt esthétique, mais aussi et peut-être principalement un intérêt commercial: traduire ou adapter au contexte local pour reproduire le succès original. Dans le contexte franco-espagnol, les exemples ne sont pas rares. "La Pulga», version de «La Puce», chanson populaire d'origine

2 Dans le contexte franco-espagnol, voir, par exemple, Jambou 2003. 
française, peut être considérée comme étant le premier succès d'adaptation musicale contemporaine dans la combinaison français-espagnol: importée des spectacles chantants parisiens - perçus comme novateurs et licencieux -, cette chanson, d'ailleurs plus salace en sa version originale, a révolutionné les cabarets et spectacles chantants madrilènes à partir de 1910 inaugurant le genre du "cuplé psiclíptico» (genre de chanson au contenu grivois interprétée par une femme) (cf. Salaün 1990: 119). Depuis, un nombre non négligeable de chansons françaises a été traduit en espagnol, ces chansons traduites étant interprétées par les artistes originaux ou par des chanteurs espagnols. Les traductions de chansons espagnoles en français semblent être moins nombreuses, pour des raisons complexes d'ordre socio-historique et économique que nous évoquerons plus avant.

Nous pouvons donc identifier quatre formes principales dans les flux musicaux transculturels: a) le réception culturelle de l'œuvre en version originale; b) la reprise musicale où les paroles originales sont remplacées par un texte totalement nouveau, et qui normalement est dépourvue de l'empreinte culturelle de l'original; c) l'appropriation de styles/genres importés selon différents degrés et suivant des stratégies musicales variées et d) la traduction et l'adaptation des paroles, moins fréquente de nos jours, mais très populaire il y a quelques décennies.

La première modalité, aujourd'hui majoritaire en raison des phénomènes divers de mondialisation et de cosmopolitisation, constituerait la première étape dans la pénétration de la transculturalité dans le domaine des musiques amplifiées. La reprise musicale ${ }^{3}$, quant à elle, serait, en général, moins marquée par le contexte de départ et relèverait d'avantage de la musicologie que des études culturelles. À notre sens, l'empreinte transculturelle la plus répandue et la plus profonde, mais aussi la moins facile à cerner, correspond probablement à l'importation ou emprunt de styles/genres musicaux dans un nouveau contexte culturel qui s'appropriera progressivement ces emprunts culturels / musicaux dans un processus de naturalisation stylistique. Tel est le cas des musiques d'origine afro-américaine - du rock au jazz en passant par le hip hop -: perçus comme étrangers dans un premier temps, ces genres ont peu à peu été incorporés aux scènes musicales nationales, en France d'abord puis en Espagne quelques années plus tard, pour intégrer aujourd'hui, à part entière, ces scènes nationales. Pour sa part, la chanson

3 La reprise est un phénomène complexe et extrêmement intéressant du point de vue de la musicologie et des études culturelles. À cet égard, voir les deux numéros consacrés au concept par la revue Volume!, Éditions Seteun, 2010, 7-1, 7-2. 
traduite se trouve à mi chemin entre la musique reçue comme élément extérieur et linguistiquement désémentisé (ou presque) et l'adaptation de la production locale aux styles importés. Face à la reprise exclusivement musicale, ce qui caractérise la traduction de chansons c'est qu'elle atteste un intérêt pour l'original dans sa composante musicale mais aussi verbale. Bien que la traduction de chansons constitue un phénomène extrêmement intéressant et complexe pour la traductologie ${ }^{4}$, l'approche retenue dans ce travail est "culturelle» et s'intéresse de préférence aux choix des chansons traduites / adaptées ainsi qu'à leur situation et à leur devenir aussi bien dans la culture de départ que dans la culture d'arrivée.

De ce point de vue, qu'il s'agisse de versions originales, de versions traduites, d'adaptations stylistiques ou de reprises musicales concrètes, toutes ces chansons transculturelles vont occuper une place bien précise dans la culture cible; une fois que ces importations s'y sont introduites, elles établissent des rapports avec les œuvres et leurs systèmes locaux, qui sont transformés en conséquence. En ce sens, il semble utile d'appliquer la théorie des polysystèmes, référée normalement au domaine des traductions littéraires, au domaine des musiques populaires. Cette théorie considère que la sélection des œuvres traduites est déterminée par la configuration du polysystème local de réception (Even-Zohar 1976: 25); ainsi, les œuvres traduites peuvent jouer des rôles différents, allant de l'innovation et la création de nouveaux modèles (esthétiques, idéologiques) lorsque le système de réception est jeune ou incomplet à, dans les polysystèmes forts, la confirmation et/ou la consolidation des valeurs déjà existantes. Bien évidemment, la situation périphérique ou dominante des œuvres traduites ou importées change au gré de l'évolution historique et de la nature des œuvres elles-mêmes. Ainsi, toute analyse des flux transculturels doit tenir compte de la configuration du polysystème de départ et du polysystème d'arrivée, de leurs rapports hiérarchiques et de force, du rôle des marchés et des modes, des interactions avec les autres systèmes impliqués et ainsi de suite. À notre sens, cette logique de relations transculturelles est applicable aux différents domaines de la culture, y compris à celui des musiques populaires.

\section{Le succès d'une «mauvaise réputation »}

Dans le contexte qui nous occupe, comme nous l'avons déjà signalé, la transculturalité «chansonnière » se fait en priorité de la France vers l'Espagne.

4 Pour une étude traductologique, voir notamment Peter Low (2005) notion de singability. 
Ceci tient, à notre sens, à la logique des relations culturelles établies entre les deux pays, et marquées, à compter du XIX ${ }^{e}$ siècle par une prééminence des modèles français en Espagne et une présence inférieure de la culture espagnole en France. Certes, les deux pays entretiennent des rapports bien trop complexes pour que nous puissions ici les explorer en détail. Or, même très schématiquement, il est possible d'affirmer que la France, au cours du $\mathrm{xx}^{\mathrm{e}}$ siècle, en dépit des graves conflits intérieurs et extérieurs qui l'ont marquée, est restée une puissance économique, politique mais aussi et surtout, une puissance culturelle qui n'a vu faiblir son empreinte symbolique qu'avec la progression parallèle du modèle américain. L'Espagne, de son côté, suite à la débâcle politique et économique du XIX ${ }^{\mathrm{e}}$ siècle, a dû faire face à un long processus de reconstruction, freiné économiquement par la guerre civile et intellectuellement par la dictature nationale-catholique, qui n'a commencé à s'ouvrir aux influences «libérales » étrangères qu'à partir du milieu des années 1950. En Espagne, l'Hexagone, même décrié par les plus conservateurs, reste le modèle étranger par excellence et sa langue continue d'être la seconde langue d'enseignement, en tant que véhicule d'une culture prestigieuse. Pour sa part, dans l'imaginaire culturel français, l'Espagne reste en général un pays de clichés, exotique et passionnel, avec une présence plutôt anecdotique dans la culture française. Dans ce contexte historicoculturel - certes, bien trop schématique -, il semble logique que les transferts se fassent du centre vers la périphérie, du modèle confirmé mais aussi plus innovateur, vers le modèle "dépassé» ou «immature» à certains égards, comme c'est notamment le cas dans le domaine des musiques populaires.

Suivant cette logique, à partir des années 1950 - la guerre et l'immédiate après-guerre ont pratiquement coupé les ponts avec l'étranger jusqu'à cette date -, les musiques populaires espagnoles, moins développées qu'en France, en Grande Bretagne ou aux Etats-Unis, se nourrissent logiquement des importations. Lorsque la majorité des jeunes Espagnols danse au son du yéyé national (assimilation/importation des modèles anglo-saxons) ou de la copla, des musiques désamorcées, en principe, de toute force contestataire, il existe tout de même une "élite» progressiste - les étudiants, les intellectuels - qui devient adepte de la chanson d'auteur française, très chic, très a la mode, qu'ils perçoivent comme très engagée.

Le cas de la présence de Brassens en Espagne s'avère très intéressant à cet égard, car il a exercé une influence claire dans la musique populaire espagnole de la deuxième moitié du $\mathrm{xx}^{\mathrm{e}}$ siècle. En effet, Brassens, mythe de la chanson française, vénéré comme poète et idole populaire nationale, 
véhicule une image idéalisée de la France... Il est à la fois local et global; «le plus français des chansonniers-poètes» (Wilmet 1992-1993: 184) et une véritable star internationale (Le Forestier, cité par Vassal 1991: 315). On perçoit ainsi une véritable tension entre le localisme absolu du personnage et l'universalité de sa réception et, ce qui est d'autant plus révélateur, la soidisant universalité des valeurs qu'il incarne.

Brassens, malgré ses réticences vis-à-vis de la traduction ${ }^{5}$, a lui-même enregistré cinq chansons en espagnol, traduites par son ami Pierre Pascal. Il n'en ai resté que trois: «La mauvaise réputation », «Le testament» et «La cane de Jeanne», un 45 tours qui n'a finalement pas été mis en vente car la maison de disques aurait eu peur de la censure franquiste. Or, l'enregistrement de ces morceaux atteste déjà l'intérêt de la maison de disques et de Brassens lui-même pour le marché musical espagnol, qu'on jugeait, en principe, apte à recevoir ces chansons. Par ailleurs, sur le plan de la réception, lorsqu'on écoute Brassens chanter en espagnol, on perçoit quelque chose d'étrange, peut-être à cause de la prononciation ou des tournures «bizarres» de quelques expressions, mais surtout parce qu'il ne s'exprime pas en français. En effet, et là il n'a pas tort, pour être lui-même, Brassens dit devoir chanter en français; sa réception est alors marquée linguistiquement et culturellement, elle n'est par conséquent pas universelle...

Bien qu'il ne se soit jamais produit en Espagne, l'empreinte de Brassens dans le pays, à partir des années 1960, est certaine; son influence est visible dans trois des modalités que l'on énonçait plus haut, à savoir: la réception directe de ses chansons - il y avait sans aucun doute un public qui écoutait Brassens en version originale -, la traduction et l'adoption de son style par des auteurs locaux. Par ailleurs, son œuvre a même fait l'objet de traductions publiées sous forme de livre (Chao 1973; García Calvo 1983) ce qui prouve bien qu'il jouissait d'un prestige culturel «savant». En ce qui concerne «l'acculturation stylistique», son influence sur les auteurs-compositeursinterprètes espagnols est évidente: sur Paco Ibáñez, sur Javier Krahe mais aussi sur Moncho Alpuente ou Joaquín Carbonnell, et en général, sur la nova cançó (Aragüez Rubio 2006: 87). Quant aux traductions interprétées par

5 «J'endurais les interprètes avec résignation. Les traducteurs finiront de m'écorcher dans tous ces idiomes, ces dialectes, ces jargons? C'est une gageure. On peut toujours faire passer dans une autre langue le sens général de la chanson. Chez moi, il est secondaire. C'est la forme qui l'emporte. Ce sont les mots, leur accouplement inattendu qui font le piquant, le vif, le sel de mes petites conneries. Je ne suis que par eux» Georges Brassens (in Radiguet 2006: 126). 
des chanteurs espagnols, les plus connues sont celles de Paco Ibáñez, Javier Krahe et Loquillo.

Paco Ibáñez est peut-être le plus célèbre des trois interprètes espagnols de Brassens, et son "disciple» avéré. En effet, c'est en suivant l'exemple du chanteur de Sète qu'il décide de mettre en musique les grands poètes espagnols comme Miguel Hernández, García Lorca, Machado ou Rafael Alberti, entre autres. Ibáñez devient bientôt une des figures artistiques de l'exil espagnol en France; symbole de la contestation politique, il contribue, en quelque sorte, à créer une certaine image de l'Espagne. En 1969, son passage à l'Olympia, où il chante pour la première fois en France «La mauvaise réputation » le consacre devant le public français. Le succès de Paco Ibáñez est, paradoxalement, d'abord français, mais bientôt ses chansons sont exportées, notamment en Espagne, où il contribue largement à la diffusion de Brassens en version originale et en version traduite, mais aussi du «style» Brassens. Lui-même, dans ses propres chansons, est entièrement débiteur de ce style Brassens; il assume à tel point ce rôle, cette conception de la création artistique, de la scénique même, qu'il est difficile de savoir dans quelle mesure le public espagnol le perçoit comme l'original au détriment de Brassens lui-même. Notons au passage que c'est en dernière instance grâce au modèle Brassens, par le truchement de Paco Ibáñez, que les grands poètes en langue espagnole ont, à leur tour, été exportés et connus du public français (et parfois aussi espagnol), ce qui prouve que les flux transculturels sont aussi bidirectionnels.

La première réception de Brassens en Espagne a donc été fortement politisée; le public progressiste utilise son œuvre et celle des autres auteurscompositeurs-interprètes ou protest singers venus de pays «libres» comme des emblèmes de la contestation contre l'ordre établi. Avec la transition démocratique, la mode des cantautores ne tarit pas, mais l'engagement politique ne semble plus être la seule priorité de la jeunesse, qui développe très rapidement d'autres types de cultures jeunes, et notamment la fameuse «movida». En 1981, au beau milieu de cette dernière, lorsque le punk et le rock font fureur, lorsque la France n'est plus le premier modèle culturel prestigieux, Javier Krahe inclut dans son premier album deux magistrales traductions de Brassens: «La tormenta» et «Marieta» («L'orage» et «Marinette» respectivement). Le public contemporain ignore souvent qu'il s'agit de traductions, ce qui atteste non seulement la réussite des processus traducteurs mais aussi l'assimilation du modèle transculturel. Or, au-delà des traductions, dans l'ensemble de son œuvre, Krahe a largement adopté 
le style Brassens: pour d'aucuns plus poète que musicien, fin humoriste, souvent accompagné d'une guitare sèche, critique incorruptible, refusant toute médiatisation, en dépit de son originalité indiscutable, c'est le Brassens espagnol par excellence. En s'intégrant dans le courant des chanteurs à texte, Krahe se marginalise par rapport aux modes et aux marchés tout en conservant une position culturelle prestigieuse. En l'absence de priorité donnée à l'engagement politique, puisque la démocratie est déjà instaurée, Krahe effectue en réalité une lecture plus «juste» de Brassens, moins médiatisée par les circonstances historiques.

Dans un style différent, un peu plus tard, Loquillo, rocker de la «troisième vague» enregistre, lui, aussi, «La mauvaise réputation» (Morir en primavera, EMI, 1988) qui, transformée en chanson rock, perd le «style» Brassens, mais lui confère aussi une deuxième jeunesse... À la fin des années 1980, la musique populaire espagnole n'en est plus à ses débuts, l'Espagne est définitivement entrée dans les courants culturels mondiaux et les influences, comme l'atteste le choix du rock comme genre, sont, avant tout, anglo-saxonnes. Dans cette version, on apprécie donc un mélange de deux modalités de transferts: d'une part, la reprise de la traduction de Pierre Pascal et l'hommage au maître Brassens, à la «tradition» des chanteurs à texte, et d'autre part, l'appropriation des styles américains, notamment du rock. Le résultat: un véritable succès où très peu reconnaissent l'original et qui, aujourd'hui même, fait partie des grands «tubes» de Loquillo.

D'autres chansons adaptées et/ou traduites confirment cette logique transculturelle entre la France, pays exportateur de prestige, et l'Espagne, pays récepteur de nouveautés. Or, le rôle tenu par les «importations » n'est pas toujours un rôle transgresseur. Tel est le cas, par exemple, de l'adaptation de «Mon homme» (Jacques Charles / Albert Willemetz / Maurice Yvain), originalement interprétée par Mistinguett, reprise par Concha Piquer (1922), puis par Sara Montiel dans la bande originale du film La violetera (1958). C'est cette dernière version qui est devenue un des grands succès de "Sarita " et que le public reconnaît aujourd'hui sans toutefois établir de lien avec l'original français. «Es mi hombre», (paroles de Jesús María de Arozamena), aujourd'hui classique du cuplé espagnol, reprend avec presque trente ans de décalage l'expression du désir féminin par celle qui deviendra une des plus grandes stars sous Franco - dont les mœurs privées contredisaient, d'ailleurs, la morale publique de l'époque. Dans ce cas, ce n'est plus la liberté politique que l'on emprunte à la culture française, mais une liberté différente, d'ordre 
individuel, sentimental et érotique ${ }^{6}$ et dont les effets sur la société sont, en principe, plus limités. En effet, la chanson récupère la tradition du cuplé gallicisme linguistique et genre musical assimilé à Paris, ce lieu mythique où surgissent nouveautés, raffinements, modes et érotismes divers. Or, le succès populaire du film, l'intronisation de Sara Montiel comme vedette du régime et l'image de la femme passionnée mais soumise, véhiculée par le texte, nous forcent toutefois à nous demander si la version de 1958 ne serait pas en fait venue confirmer le statu quo idéologique de l'époque sous prétexte d'évoquer une certaine atmosphère "parisienne» et libérale. Les «nouveautés» venues de la "ville lumière» ne sont plus innovantes - c'est une chanson vieille de 30 ans! - mais jouent plutôt un rôle conservateur sous l'apparence fallacieuse de la modernité étrangère.

\section{Conclusions}

La transculturalité dans le domaine des musiques populaires, comme nous l'avons vu dans les exemples, regroupe un ensemble de procédés de translation musicale et verbale dont la logique relève des rapports complexes (socio-économiques, idéologiques, esthétiques, symboliques) existant entre les polysystèmes en contact. Deux stratégies principales régissent ces transferts culturels. D'une part, une stratégie d'innovation: le polysystème perçu comme prestigieux et/ou moderne exporte ses œuvres dans un polysystème périphérique dont les forces innovantes réclament des nouveautés capables de faire évoluer / progresser leurs propres systèmes. D'autre part, une stratégie de conservation: le polysystème de réception emprunte au polysystème extérieur des objets confirmant des valeurs préexistantes, renforçant alors la doxa établie. Logiquement, c'est la première des stratégies qui produit les effets les plus visibles sur la culture de réception, qui entraîne les changements. Ainsi, dans l'Espagne franquiste, l'apparition et l'assimilation progressive de nouveaux objets culturels venus de l'extérieur - dont la musique populaire - contribuera à faire sortir le pays de son isolement, à créer de nouveaux sons, de nouveaux discours, de nouvelles esthétiques, qui seront par la suite assimilés pleinement par le polysystème local, comme l'attestent les versions plus récentes des chansons de Brassens et que Krahe, Loquillo et Ibáñez continuent d'interpréter dans leurs concerts. De leur côté, «Es mi hombre» ou les versions espagnoles des

6 Une lecture féministe des paroles et des performances respectives des interprètes serait très intéressante mais elle déborde l'objet du présent travail. 
«tubes» français ${ }^{7}$ témoignent de la stratégie conservatrice, dont les effets sont, en général, limités à une modernité factice.

Les transformations culturelles et sociales résultent donc, en partie, des flux transnationaux qui voyagent notamment du centre vers la périphérie mais aussi dans le sens inverse comme l'atteste le rôle de certains artistes comme Paco Ibáñez dans la diffusion de la culture espagnole en France. Ainsi, dans les années 1910, l'Espagne a importé l'érotisme et le "dévergondage» féminin des cafés-concerts parisiens, contribuant ainsi à la modernisation des spectacles et des mœurs d'une certaine classe socio-culturelle. Dans les années 1960 et 1970, la culture et la musique populaire espagnoles avaient besoin de Brassenset de la chanson française "poétique» comme révulsif esthétique et politique; c'est alors que la chanson à texte, inspirée du modèle français et américain, est devenue une arme pour la contestation. Toutefois, à partir de la transition et de la «modernisation » de l'Espagne, le style Brassens et des chanteurs à texte, a cessé de constituer un facteur d'innovation, mais a su conserver son prestige.

Parallèlement, la moindre présence des musiques espagnoles sur la scène française du $\mathrm{xx}^{\mathrm{e}}$ et $\mathrm{xxI}^{\mathrm{e}}$ siècles constituent, à notre sens, la preuve que le prestige, et donc l'influence, de la culture espagnole en France est bien limité... En ce sens, et de façon significative, les musiques espagnoles ayant le plus d'écho en France sont celles que l'on assimile aux «musiques du monde» - flamenco et andalouses, auxquelles s'ajoutent, en dépit des différences fondamentales, les musiques latines. Ceci répond bien aux nouvelles logiques transculturelles à l'œuvre sur les marchés des pays postindustrialisés depuis les années 1980, qui font résider le rôle d'innovation dans les musiques excentrées. En effet, c'est désormais la "world musique», la "périphérie» musicale qui, récupérée par le système prestigieux ou dominant, vient à «révolutionner» ce dernier, même s'il s'agit, certes, d'une révolution «tranquille» et largement contrôlée par les polysystèmes d'accueil.

7 Vázquez Montalbán, dans son Cancionero General del Franquismo (2000), ne tient compte que des chansons traduites dans l'Espagne franquiste, des chansons sentimentales, dépourvues en principe, de toute charge contestataire 


\section{Bibliographie}

Aragüez Rubio, C. 2006, «La nova cançó catalana: génesis, desarrollo y trascendencia de un fenómeno cultural en el segundo franquismo ", Pasado y memoria: Revista de historia contemporánea, $\mathrm{n}^{\circ} 5$ : 81-98.

Brassens, G., 1983, 19 canciones con versión para cantar, éd. et traduction de García Calvo, A., Madrid, Lucina.

Chao, R. 1973, Georges Brassens, Madrid, Júcar.

Even-Zohar, T., 1976, "The Position of Translated Literature Within the Literary Polysystem», conference pronnoncée à l'occasion du Leuven International Symposium "Literature and Translation: New Perspective in Literary Studies", The Catholic University of Leuven, 27-30 avril 1976.

Gabriel y Galán, J.A., 1982, «Brassens y la cofradía de los desconocidos», El País, 21/11/1982.

Jambou, L. (éd.), 2003, La musique entre France et Espagne: interactions stylistiques. I, 1870-1939, Paris, Presses de l'Université Paris-Sorbonne.

Katz, M., 2004, Capturing Sound: How Technology Has Changed Music, Ewing, University of California Press.

Low, P., 2005, "The Pentathlon Approach To Translating Songs» in Song and significance, ed. Dinda L. Gorlée, Amsterdam-New York, Rodopi: 185-212.

Radiguet, Ch., 2006, Brassens à la lettre, Paris, Delanoë.

Salaün, S., 1990, El cuplé (1900-1936), Madrid, Espasa.

Vassal, J., 1991, Brassens ou la chanson d'abord, Paris, Albin Michel.

Vázquez Montalbán, M., 2000, Cancionero general del franquismo (1939-1975), Barcelona, Crítica.

Vertovec, S. et Cohen, R. (éds), 2002, Conceiving cosmopolitanism: theory, context and practice, Oxford, Oxford University Press: 1-22.

Wilmet, M., 1992-1993, "Postface», Equivalences, 22/1-2 et 23/1: 183-189.

\section{Discographie}

Georges Brassens, 2009, Elle est à toi cette chanson. L'intégrale, Mercury. Javier Krahe, Joaquín Sabina et Alberto Pérez, 1981, La mandrágora, CBS. Loquillo y los Trogloditas, 1988, Morir en primavera, Hispavox.

Mistinguett, 1916, «Mon homme».

Paco Ibáñez, 1979, Paco Ibáñez canta a Georges Brassens, A Flor de tiempo/Universal Music.

Sara Montiel, 1958, «Es mi hombre». 